\title{
Wall Embedding Type High Safe Micro Control System Research
}

\author{
Haiying Zhang ${ }^{1 \mathrm{a}}$, Xiangyan $\mathrm{Yu}^{2 \mathrm{~b}^{*}}$ and Zhenjun $\mathrm{Chen}^{1 \mathrm{c}}$ \\ ${ }^{1}$ Qingdao Binhai University, Qingdao, China, 266555 \\ ${ }^{2}$ Qingdao Qian wan Container Terminal Co., Ltd., Qingdao, China, 266550 \\ a64029205@qq.com, b8311556@qq.com, czhanghy_ok@sina.com
}

\begin{abstract}
Keywords: Wall safes safe embedded advanced; Micro-control-system; LC246 alarm module; PIC16F877 Amicrocontroller
\end{abstract}

\begin{abstract}
This paper mainly studies the wall mounted high safe micro control system. The use of a new control system make the safe is safer, more reliable. The safe has built-in microcomputer control chipPIC16F877A, realizes the password control, automatic lock, an indoor alarming and monitoring network and a series of functions, the thief cannot start. The apparatus is convenient for household use, and is convenient for residential property and Public Security Bureau network monitoring, has great social benefit and development foreground.
\end{abstract}

\section{Introduction}

The sixties and seventies of the 20th century because of the ever-changing semiconductor technology, the industry developed electronic locks, electronic locks are widely used in various types of safe products. After the turn LED, LCD digital display for safe, user demand for fire protection has also spawned a variety of fire protection products, fingerprint scan recognition technology development to promote the use of the fingerprint lock in the safe, and safe product categories when the most simple functions to develop to theft, fire, burglar / fire, certain types of magnetically shielded, home, commercial, hotel, etc.. A number of safe is not suitable for embedded walls, large, bulky weight, the safe system of internal controls in this study a wall embedded, the space-saving, safe

\section{Wall Embedded Micro-control System Safe}

Micro-control Circuit. The circuit consists of a central control unit, the keyboard input unit, liquid crystal display unit, alarm unit, motor drive unit, the signal detection unit, modulation and demodulation unit, power supply circuit of several parts of the circuit components

Setting the external interface with the motherboard 12v AC power input interface Jac12v, indoor Burglary, Fire interfaces Jhm, for help button interface Jhp, network line interface Jline, online programming and debugging interfaces Jtext.

Set the board's internal interface has four $1.2 \mathrm{v}$ rechargeable battery backup power interface Jdc, 16 key keyboard interface Jkey, LCD liquid crystal display interface Jmonitor, speaker interface, Jsp, LED indicator Interface Jled, motor control interface Jmd, lock body position sensor Interface Jcg. Refer to Figure 1 control circuit diagram

Central Control Unit. Master chip IC1, the Microchip's PIC16F877A microcontroller as the basic module $8 \mathrm{Kx} 14$ MCU-bit Flash program memory, 512 bytes of special and common unit of data storage, 256x8-bit non-volatile E2PROM data memory, arithmetic logic unit ALU, 5 input 33 output ports of I/O pins, configured three multifunction timer modules, embedded in a more powerful watchdog timer, with a strong low-pressure line debugging and programming; the dedicated single-chip synchronous serial port module with SSP, universal Synchronous / asynchronous Receiver Transmitter USART, parallel Slave port PSP, configure two distinctive capture/Compare/PWM module CPP1 and CPP2, embedded in a 10-bit resolution A/D converter $\mathrm{ADC}[1]$.

PIC16F877A total of five input and output ports A, B, C, D, E. Which has six port A pins RA0 RA5; Port B has eight pins RB0 RB7; eight port C pins RC0 RC7; eight port D pins RD0 RD7; 
port E has three arguments pin RE0 RE2. These pins have different functions

This design uses Flash memory storage control program, through the debug interface JTEXT line input and debugger, the use of E2PROM memory storage safe passwords, telephone numbers and Internet address of the alarm by a large number of $\mathrm{I} / \mathrm{O}$ interface to control an external circuit through the USART module automatic multi-machine network alarm.

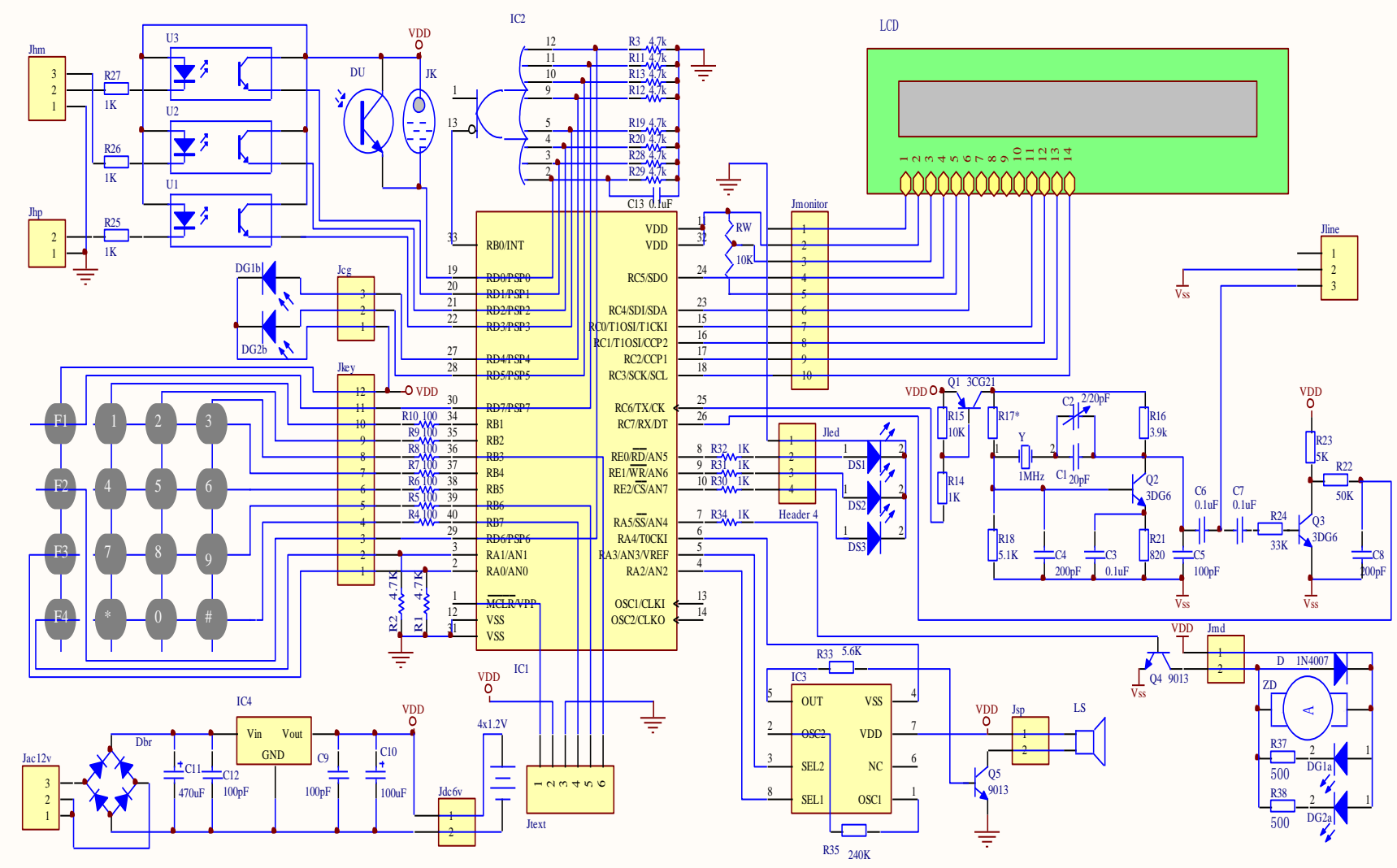

Figure 1. Schematic control

Keyboard Input Unit. 16-key membrane keyboard connected to PIC16F877A via interfaces Jkey the RB1 RB7, RA0 RA1, RD6 RD7 pins. Which RB1 RB7 matrix composed of four lines of three connected 1,2,3,4,5,6,7,8,9,0, *, \# twelve keys, RB4 RB7-change interrupt function, when the press any key to interrupt when notified by the CPU processing; RD7, RD6 and VDD connected to the function keys F1 and F2, when press F1 or F2 key, RD7 RD6 to generate a high level or through the gate by the IC2 or IC1's external interrupt request input terminal INT send a request to the CPU; RA0, RA1 and VDD connected to the function keys F3 and F4 on the two key functions without interruption request, with the principal and other key operations.

LCD Display Unit. LCD Module LCD, you can display a letters, numbers, symbols and other data point formation module HD44780. A total of 14 pins, pin No. 1 is the power to VSS, No. 2 pin is the power supply VDD, No. 3 pin power the LCD display driver, No. 4 pin is the register select signal RS, No. 5 pin to read and write select signal R/W, No. 6 pin enable signal E, 7 to 14 pins for the three-state data bus DB0 DB7 [2].

The design of RC0 RC3 PIC16F877 and HD44780 data bus connected to the high four DB4 DB7, a two-input eight-bit data; RC4 and HD44780 connected to the enable signal E; RC5 and HD44780 connected to the RS, is used to select data register and the instruction register. Related lead is connected through an interface $\mathrm{J}$ monitor.

Alarm Output Unit. Simulated using four-LC246 IC IC3 as an alarm sound alarm sound source, LC246 Manifold 8-pin plastic dual in-line hardware package, the pin functions: 1 oscillator input pin OSC1, 2 feet oscillator output end OSC2, 1,2-pin external oscillator resistor; 3 feet for the second election the sound side SEL2; 4 pin negative power supply VSS; 5 feet as an alarm signal output 
terminal oUT; the first 6 feet of empty legs NC; 7 pin positive power supply VDD; 8 feet is the first election the sound side SEL1. When SEL2 then high, output short alarm sound; when SEL2 vacant, SEL1 fire engine access high output sound, SEL1 low-level output when the ambulance sound, SEL1 output is also vacant police car sound [3] (P21).

Because of this design is the PIC16F877A's RA4 pin drain output pin RA4 direct control LC246 with the negative terminal VSS; RA2 control LC246 with the sound side of SEL1 election; RA3 control LC246 with the sound side of SEL2 election. LC246 output OUT through a resistor R33 control transistor Q5, the alarm sounds sent through the interface Jsp speaker LS

Motor Drive Unit. The RA5 pin by the PIC16F877A connected transistor Q4 through resistor R34 to the base, collector received Jmd interface, the interface through Jmd ZD on the motor, which play a protective role of the diode D, LED DG1a detection device to lock in place to provide light, light tube DG2a detection devices in place for the lock off to provide light.

Signal Detection Unit. Using PIC16F877A eight port D pins RD0 RD7 enter the amount of various types of testing, or by the reverse gate IC2 output terminal to generate a falling edge through RB0/INT interrupt request to the CPU. RD0 to receive information about the local police, when JK mercury switches are turned on or photodiode light irradiation DU will enter high; RD1 indoor theft alarm used to receive information through the optical isolator U3 device through the interface Jhm and indoor Pirates of the police unit to contact; RD2 indoor burglary to receive information on the device through the optical isolator U2 Jhm through the interface unit and indoor fire contact; RD3 U1 through the optical isolator for help with the interface Jhp and external buttons connected, easy to to seek help information to the network; RD4 directly through the interface Jcg and photodiode DG1b connected transmission lock in place information; RD5 directly through the interface Jcg and photodiode DG2b connected, the transmission locks in place the relevant information; RD6 and RD7 for keyboard input, not repeat them.

Modem Unit. In order to achieve automatic networking and more, this design uses a PIC16F877A's internal USART module serial communication, data transfer from the RC6, RC7 of the data receiver. In order to achieve the use of ordinary telephone lines and reliable transmission of information, design a modem, as shown.

Data transmission: PIC16F877A pin RC6 output from the digital signal into the PNP type switch the base of Q1, thereby keying the transistor Q2, the crystal oscillator Y, resistors R16 R18 R21 and capacitor $\mathrm{C} 1 \sim \mathrm{C} 6$ oscillation composed device. When RC6 output low, transistor Q1 turns on, the oscillator work, the capacitor $\mathrm{C} 6$ to the interface, J line transmission frequency information; when RC6 output low, the transistor Q1 off, the oscillator stops, J line is no high-frequency information. Thus the transmission of binary information. Thus the signal modulation.

Data reception: from Interface $\mathrm{J}$ line came through the modulation signal coupling capacitor $\mathrm{C} 7$ to the base of transistor Q3 through the detection transistor Q3, resistor R22, R23 and capacitor C8 filtering, modulation of the signal has been restored. Demodulated signal directly to the serial port of the receiver RC7, when, after receiving a message sent to the CPU interrupt request

Power Circuit. Main Power: 220V mains transformer reduced by 12V, send interface, Jac, by the heap Dbr bridge rectifier, filter capacitor C11, the LM7805 regulator IC4 into the 5V DC power supply to the main circuit. Standby Power: 4 AA $1.2 \mathrm{~V}$ rechargeable batteries provide backup via the interface Jdc.

\section{To Control Program Flow}




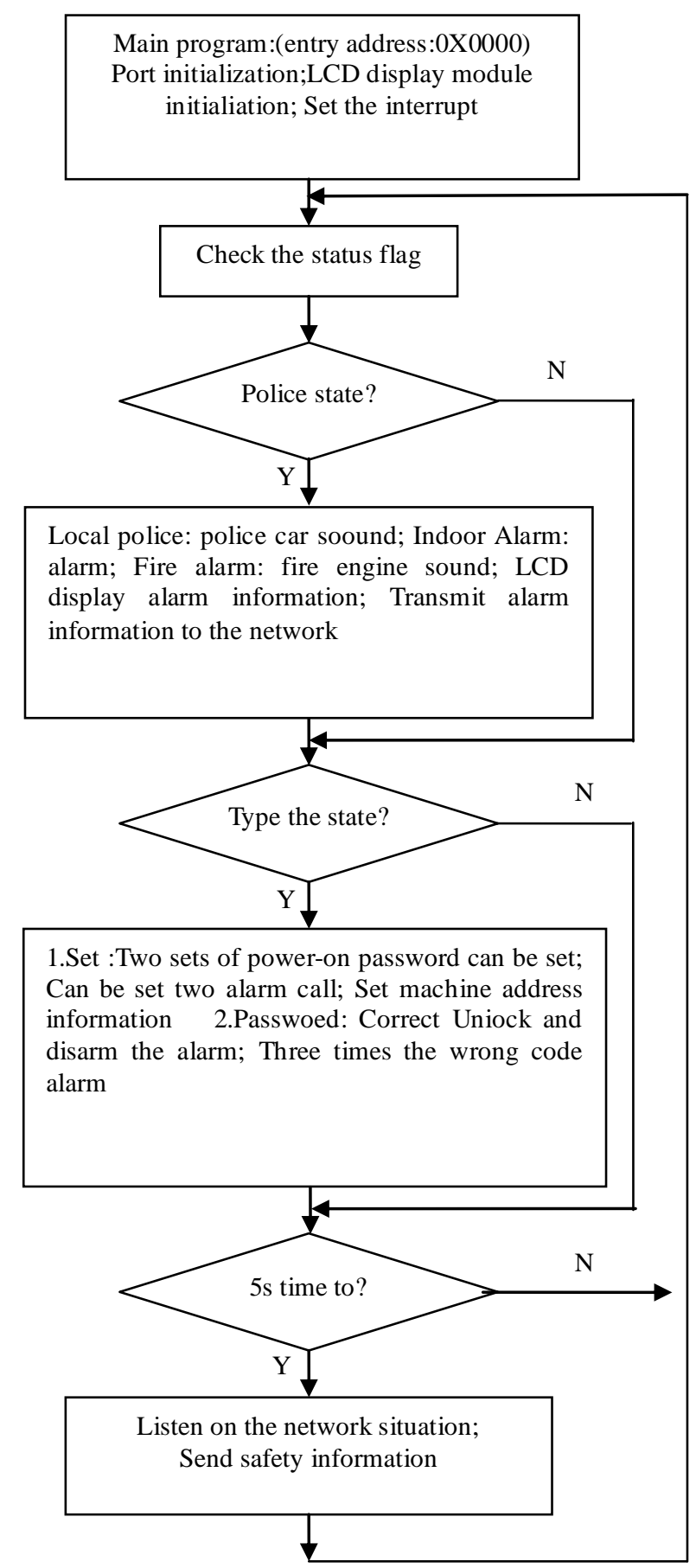

Figure 2. The main program flow

\section{The Structural Characteristics of the Wall Embedded Safe}

a) interior $24 \mathrm{~cm}$ reinforced concrete box embedded within the wall, the wall surface and in the same plane, covered with ornaments, no space, strong and good.

b) use of automatic electronic locks, the number of passwords over 10 billion combinations, thieves can not decipher.

c) the use of bold multi Fangqiao Men bolt, bolt diameter 24-30mm, controlled by the agency automatic.

d) the use of advanced membrane keypad, a waterproof, moisture-proof, wear-resistant properties. e) facade using $3-5 \mathrm{~mm}$ thickness cold rolled steel, doors and door frames with more closely.

f) the wrong code three times, vibration, flood, fire alarm.

g) The machine can provide LCD or LED display and alarm sound, between residential 
properties and residents can monitor networking with each other. With mobile phones, telephone networking alarm matched.

\section{Conclusions}

In this paper, wall safe micro-embedded advanced control system carried out a detailed design, the design control procedures using Flash memory storage, automatic multi-machine network alarm, control system design is simple and sensitive, safe to overcome the shortcomings of the past. The common market with a variety of components, convenient operation, safe for the future development of similar micro-control system to provide a reference

\section{References}

[1] Li rong zheng,etc.PIC Microcontroller Theory and Applications[M], Beijing: Beijing Aerospace University Press, (2005), (1):16-20

[2] Tang jing nan,etc. PIC microcontroller-based and application [M], Beijing: People's Posts and Telecommunications Press, (2006), (6):311-317

[3] Chen you qing, etc. alarm and alarm IC production instance [M], Beijing: People's Posts and Telecommunications Press, 1996, (2): 21

[4] Zhang fang, xiong dong, Liu xiao ming, etc. DMF-based and multi-code spread spectrum Yuan cumulative sentences capture method [J]. The world's scientific and technological research and development, (2010), 32 (3): 282-284.

[5] Hu hui, Li ye zi, Li guo hong, etc. safe encrypted IC card controller design, Journal, (2001), No. 6.

[6] Liu hai song, Wu Jie long, Guo zhao you.Based on the ship set VICTOR module embedded SBC power control system design. Journal, (2009), the first phase.

[7] Liu jia, Li yun ze, Chang jing, Sheng jiang. Tiny research satellite thermal control system status and development trend. Spacecraft Environment Engineering, February (2011).

[8] Huang hong, Zhang shao jun. PLC synchronous motor excitation system in the application. Equiment Manufactoring Technology NO.11. (2007).

[9] Chen wei. DC servo machine tool spindle of the PLC system. Journal, (2003) Ninth.

[10]Zhu shi xue. Reversible speed circulation controlled DC servo system applied research of manufacturing automation, March (2009)

[11] Huang wei, Hu qing long. Manipulator PLC control system design mechanical and electrical engineering, (2008) 